\title{
Einstein-Riemann Gravity on Deformed Spaces
}

Julius WESS $\dagger^{1} \dagger^{2} \dagger^{3}$

$\dagger^{1}$ Arnold Sommerfeld Center for Theoretical Physics Universität München, Theresienstr. 37, 80333 München, Germany

E-mail:wess@theorie.physik.uni-muenchen.de

$\dagger^{2}$ Max-Planck-Institut für Physik, Föhringer Ring 6, 80805 München, Germany

$\dagger^{3}$ Universität Hamburg, II Institut für Theoretische Physik and DESY, Luruper Chaussee 149, 22761 Hamburg, Germany

Received October 27, 2006, in final form November 28, 2006; Published online December 11, 2006 Original article is available at http://www.emis.de/journals/SIGMA/2006/Paper089/

\begin{abstract}
A differential calculus, differential geometry and the E-R Gravity theory are studied on noncommutative spaces. Noncommutativity is formulated in the star product formalism. The basis for the gravity theory is the infinitesimal algebra of diffeomorphisms. Considering the corresponding Hopf algebra we find that the deformed gravity is based on a deformation of the Hopf algebra.
\end{abstract}

Key words: noncommutative spaces; deformed gravity

2000 Mathematics Subject Classification: 83C65; 81T75; 58B34

This article is based on common work with Paolo Aschieri, Christian Blohmann, Marija Dimitrijević, Frank Meyer and Peter Schupp [1, 2].

\section{Introduction}

Gravity theories and differential geometry have been developed on differential manifolds where the functions form an algebra by pointwise multiplication:

$$
\mu\{f \otimes g\}=f \cdot g .
$$

In this lecture I want to show that these theories can be generalized by deforming this product. There are many deformations of the pointwise product to a star product $[3,4,5,6]$; the simplest and most discussed is the Moyal-Weyl product [7, 8]:

$$
\mu_{\star}\{f \otimes g\} \equiv f \star g=\mu\left\{e^{\frac{i h}{2} \theta^{\rho \sigma} \partial_{\rho} \otimes \partial_{\sigma}} f \otimes g\right\},
$$

where $\theta^{\rho \sigma}$ is constant antisymmetric matrix. This product can be shown to be associative but it is not commutative. It is defined for $C^{\infty}$ functions in general as a formal power series in $h$. Evaluated on the functions $x^{\mu}$ and $x^{\nu}(1)$ yields:

$$
x^{\mu} \star x^{\nu}-x^{\nu} \star x^{\mu} \equiv\left[x^{\mu \star}, x^{\nu}\right]=i \theta^{\mu \nu} .
$$

These, mathematically, are the canonical commutation relations of quantum mechanics but here we postulate them for the configuration space.

${ }^{\star}$ This paper is a contribution to the Proceedings of the O'Raifeartaigh Symposium on Non-Perturbative and Symmetry Methods in Field Theory (June 22-24, 2006, Budapest, Hungary). The full collection is available at http://www.emis.de/journals/SIGMA/LOR2006.html 
A differential calculus on noncommutative spaces has been developed [9, 10, 11]. Considering differentiation as a map from the space of functions to the space of functions:

$$
\partial_{\rho}: f \mapsto \partial_{\rho} f
$$

it can be generalized to an algebra map.

Recognizing that $f \star g$ is a function, again one finds the deformed Leibniz rule:

$$
\partial_{\rho}(f \star g)=\left(\partial_{\rho} f\right) \star g+f \star\left(\partial_{\rho} g\right)+f\left(\partial_{\rho} \star\right) g .
$$

In the case of the Moyal-Weyl product the $\star$ operation is $x$-independent and we obtain the usual Leibniz rule. To indicate that the derivative now is a map from the deformed algebra of functions to the deformed algebra of functions we denote it by $\partial^{\star}$

$$
\partial_{\rho}^{\star} f \equiv \partial_{\rho} f, \quad \partial_{\rho}^{\star}(f \star g)=\left(\partial_{\rho}^{\star} f\right) \star g+f \star\left(\partial_{\rho}^{\star} g\right) .
$$

These equations establish a well-defined differential calculus on the deformed space of functions. They allow us to consider $\partial_{\rho}^{\star}$ as a linear operator with the properties:

$$
\partial_{\rho}^{\star} \partial_{\sigma}^{\star}=\partial_{\sigma}^{\star} \partial_{\rho}^{\star}
$$

and

$$
\partial_{\rho}^{\star} f=\left(\partial_{\rho}^{\star} f\right)+f \star \partial_{\rho}^{\star}=\left(\partial_{\rho} f\right)+f \star \partial_{\rho} .
$$

The following treatment of deformed differential geometry will be based on the equations (2), (4) and (5). It is only the equation (3) that defines the ordinary derivative of a function that has to be used as an a priori input. The generalization to the deformed situation is essentially algebraic of nature.

\section{Differential operators}

We now consider the extension of the algebra of functions (deformed or undeformed) by the algebra of derivatives. From the Leibniz rule (5) follows that there is a basis where the derivatives are all at the right hand side of the functions. An element of the extended algebra in this basis we call a differential operator [1].

Undeformed:

$$
\mathcal{D}_{\{d\}}=\sum_{r \geq 0} d_{r}^{\rho_{1} \ldots \rho_{r}} \partial_{\rho_{1}} \cdots \partial_{\rho_{r}}
$$

Deformed:

$$
\mathcal{D}_{\{d\}}^{\star}=\sum_{r \geq 0} d_{r}^{\rho_{1} \ldots \rho_{r}} \star \partial_{\rho_{1}}^{\star} \cdots \partial_{\rho_{r}}^{\star} .
$$

A differential operator is characterized by the coefficient functions $d_{r}$. This is indicated by $\{d\}$. We shall frequently omit this indication and write $\mathcal{D}$ for a differential operator, with coefficient function $d_{r}$ and $\mathcal{D}^{\prime}$ for $d_{r}^{\prime}$.

Differential operators can be multiplied using the algebraic properties (1) or (2) in the deformed case and the relations (4) and (5).

The product can always be expressed in terms of differential operators by reordering it with the help of the Leibniz rule. In this sense the differential operators form an algebra in both cases, deformed and undeformed. 
There is a map of the operators $\mathcal{D}$ to the operators $\mathcal{D}^{\star}$ that is an algebra morphism

$$
\mathcal{D} \mapsto \mathcal{D}^{\star} .
$$

To define this map we let the differential operators act on a function $g$ :

$$
\mathcal{D} \triangleright g=\sum_{r \geq 0} d_{r}^{\rho_{1} \ldots \rho_{r}}\left(\partial_{\rho_{1}} \cdots \partial_{\rho_{r}} g\right) \quad \text { or } \quad \mathcal{D}^{\star} \triangleright^{\star} g=\sum_{r \geq 0} d_{r}^{\rho_{1} \ldots \rho_{r}} \star\left(\partial_{\rho_{1}}^{\star} \cdots \partial_{\rho_{r}}^{\star} g\right) \text {. }
$$

We now ask for an operator that it, when acting on $g$, maps $g$ to the same function that we obtain by acting with the operator $\mathcal{D}$ on $g$. This $\star$-operator we call $X_{\mathcal{D}}^{\star}$

$$
X_{\mathcal{D}}^{\star} \stackrel{\star}{\triangleright} g=\mathcal{D} \triangleright g
$$

Because $X_{\mathcal{D}}^{\star} \stackrel{\star}{\triangleright} g$ is a function we can apply $X_{\widetilde{\mathcal{D}}}^{\star}$ once more:

$$
X_{\widetilde{\mathcal{D}}}^{\star} \stackrel{\star}{\triangleright}\left(X_{\mathcal{D}}^{\star} \triangleright g\right)=\left(X_{\widetilde{\mathcal{D}}}^{\star} \star X_{\mathcal{D}}^{\star}\right) \stackrel{\star}{\triangleright} g
$$

The left hand side can also be evaluated by using (6) consecutively:

$$
X_{\widetilde{\mathcal{D}}}^{\star} \stackrel{\star}{\triangleright}\left(X_{\mathcal{D}}^{\star} \stackrel{\star}{\triangleright} g\right)=X_{\widetilde{\mathcal{D}}}^{\star} \stackrel{\star}{\triangleright}(\mathcal{D} \triangleright g)=\widetilde{\mathcal{D}} \mathcal{D} \triangleright g=X_{\widetilde{\mathcal{D}} \mathcal{D}}^{\star} \stackrel{\star}{\triangleright} g .
$$

We conclude:

$$
X_{\widetilde{\mathcal{D}}}^{\star} \star X_{\mathcal{D}}^{\star}=X_{\widetilde{\mathcal{D}} \mathcal{D}}^{\star}
$$

Multiplying $g$ pointwise with a function $f$ forms a subalgebra of $\mathcal{D}$. We shall now construct the operator $X_{f}^{\star}$ explicitly for this case starting from (1):

$$
f \cdot g=\mu\{f \otimes g\}=\mu\left\{e^{\frac{i h}{2} \theta^{\rho \sigma} \partial_{\rho} \otimes \partial_{\sigma}} e^{-\frac{i h}{2} \theta^{\rho \sigma} \partial_{\rho} \otimes \partial_{\sigma}} f \otimes g\right\}=\mu_{\star}\left\{e^{-\frac{i h}{2} \theta^{\rho \sigma} \partial_{\rho} \otimes \partial_{\sigma}} f \otimes g\right\} .
$$

More explicitly:

$$
X_{f}^{\star}=\sum_{r \geq 0} \frac{1}{r !}\left(-\frac{i}{2}\right)^{r} \theta^{\rho_{1} \sigma_{1}} \cdots \theta^{\rho_{r} \sigma_{r}}\left(\partial_{\rho_{1}} \cdots \partial_{\rho_{r}} f\right) \star \partial_{\sigma_{1}}^{\star} \cdots \partial_{\sigma_{r}}^{\star} .
$$

This operator has the properties:

$$
X_{f}^{\star} \star g=f \cdot g \quad \text { and } \quad X_{f}^{\star} \star X_{g}^{\star}=X_{f g}^{\star} .
$$

Deformed gauge theories $[12,13,14,15]$ are based on these operators.

The algebra of functions with pointwise multiplication is mapped into an algebra of deformed differential operators.

The algebra of diffeomorphisms is generated by vector fields

$$
\begin{aligned}
& \xi=\xi^{\mu}(x) \partial_{\mu}, \\
& {[\xi, \eta]=\left(\xi^{\mu}\left(\partial_{\mu} \eta^{\rho}\right)-\eta^{\mu}\left(\partial_{\mu} \xi^{\rho}\right)\right) \partial_{\rho}=(\xi \times \eta)^{\rho} \partial_{\rho}=\xi \times \eta .}
\end{aligned}
$$

The commutator of two vector fields is a vector field again. This is not the case for the star commutator because the $\star$-product of two functions does not commute. The differential operators $X_{\xi}^{\star}$, however, will form an algebra under the star commutator:

$$
\left[X_{\xi}^{\star}, X_{\eta}^{\star}\right]=X_{\xi \times \eta}^{\star}
$$

This follows from (7). 
The operator $X_{\xi}^{\star}$ is easily constructed starting from (2)

$$
\xi \triangleright g=\xi^{\mu} \partial_{\mu} \triangleright g=\xi^{\mu}\left(\partial_{\mu} g\right)=X_{\xi^{\mu}}^{\star} \star \partial_{\mu}^{\star} \stackrel{\star}{\triangleright} g
$$

Thus, we find

$$
X_{\xi}^{\star}=X_{\xi^{\mu}}^{\star} \star \partial_{\mu}^{\star} .
$$

Again the usual algebra of diffeomorphisms is mapped into a subalgebra of the algebra $\mathcal{D}^{\star}$

$$
\xi \mapsto X_{\xi}^{\star}, \quad\left[X_{\xi}^{\star}, X_{\eta}^{\star}\right]=X_{\xi \times \eta}^{\star} .
$$

This is the starting point for the construction of a tensor calculus on tensor fields.

\section{Tensor fields}

The classical theory of gravity is based on invariance under coordinate transformations. This leads to the concept of scalar, vector and tensor fields that transform under general coordinate transformation as follows

$$
\begin{array}{ll}
\text { scalar: } & \delta_{\xi} \phi(x)=-\xi \phi, \\
\text { covariant vector: } & \delta_{\xi} V_{\mu}(x)=-\xi V_{\mu}-\left(\partial_{\mu} \xi^{\rho}\right) V_{\rho}, \\
\text { contravariant vector: } & \delta_{\xi} V^{\mu}(x)=-\xi V^{\mu}+\left(\partial_{\rho} \xi^{\mu}\right) V^{\rho}
\end{array}
$$

and so on.

The concept of coordinate transformations is difficult to generalize to deformed spaces, but the transformation laws of fields are representations of the infinitesimal diffeomorphism algebra that we know how to deform. Thus, we will postulate the following transformation laws for the deformed algebra of diffeomorphisms

$$
\begin{aligned}
& \delta_{\xi}^{\star} \phi(x)=-X_{\xi}^{\star} \stackrel{\star}{\triangleright} \phi, \\
& \delta_{\xi}^{\star} V_{\mu}(x)=-X_{\xi}^{\star} \stackrel{\star}{\triangleright} V_{\mu}-X_{\left(\partial_{\mu} \xi^{\rho}\right)}^{\star} \stackrel{\star}{\triangleright} V_{\rho}, \\
& \delta_{\xi}^{\star} V^{\mu}(x)=-X_{\xi}^{\star} \stackrel{\star}{\triangleright} V^{\mu}+X_{\left(\partial_{\rho} \xi^{\mu}\right)}^{\star} \stackrel{\star}{\triangleright} V^{\rho}
\end{aligned}
$$

and so on.

To construct Lagrangians we have to know how the $\star$-product of fields transforms. These products should transform as tensor fields again. E.g., the $\star$-product of two scalar fields should transform as a scalar field again

$$
\delta_{\xi}^{\star}(\phi \star \psi)=-X_{\xi}^{\star} \stackrel{\star}{\triangleright}(\phi \star \psi) .
$$

A lengthy but direct calculation shows that this is identical to:

$$
\delta_{\xi}^{\star}(\phi \star \psi)=-\mu_{\star}\left\{\mathcal{F} \Delta(\xi) \mathcal{F}^{-1} \phi \otimes \psi\right\},
$$

where $\Delta(\xi)$ is the usual comultiplication

$$
\Delta(\xi)=\xi \otimes 1+1 \otimes \xi
$$

and therefore an element of the $\otimes$ tensor product. $\mathcal{F}$ is called a twist and it is an element of the tensor product

$$
\mathcal{F}=e^{-\frac{i h}{2} \theta^{\rho \sigma} \partial_{\rho} \otimes \partial_{\sigma}} .
$$


The right hand side of (9) and (10) can be calculated in a power series expansion in $h$ and will be found to be the same.

The advantage of the expression (10) is that it links to the formalism of deforming Hopf algebras by twists $[16,17,18,19]$. Many results are known there. We first have to establish that $\mathcal{F}$ really satisfies the conditions for a twist [2]. For (11) it is the case. Then we can use the twist to deform the Leibniz rule for arbitrary tensor fields. The procedure is as follows:

First consider the coproduct for the undeformed transformations

$$
\Delta\left(\delta_{\xi}\right)=\delta_{\xi} \otimes 1+1 \otimes \delta_{\xi},
$$

where the variations $\delta_{\xi}$ are expressed by differential operators such that

$$
\delta_{\xi}(\phi \otimes \psi)=\left(\delta_{\xi} \phi\right) \otimes \psi+\phi \otimes\left(\delta_{\xi} \psi\right)
$$

for any two tensor fields $\phi$ and $\psi$. This coproduct can be twisted

$$
\Delta_{\mathcal{F}}\left(\delta_{\xi}\right)=\mathcal{F} \Delta\left(\delta_{\xi}\right) \mathcal{F}^{-1} \text {. }
$$

Finally the Leibniz rule becomes

$$
\delta_{\xi}^{\star}(\phi \star \psi)=\mu_{\star}\left\{\Delta_{\mathcal{F}}\left(\delta_{\xi}\right) \phi \otimes \psi\right\} .
$$

This is not limited to scalar fields but covers the $\star$-product of all tensor fields.

Finally we can convince ourselves that this Leibniz rule has the properties demanded at the beginning of this chapter, i.e. that $\star$-products of tensor fields transform as tensor fields.

Equation (12) allows us now to consider $\star$-products of fields and to determine their transformation properties.

As a Hopf algebra the algebra of infinitesimal diffeomorphisms is deformed!

\section{Einstein-Hilbert gravity}

The Einstein-Hilbert theory of gravity can now be constructed following its presentation in a text book.

\subsection{Covariant derivatives}

The covariant derivative of a tensor field should again transform as a tensor field. This can be done with the help of a connection $\Gamma$. For a covariant vector field:

$$
D_{\mu} \stackrel{\star}{\triangleright} V_{\nu}=\partial_{\mu}^{\star} \stackrel{\star}{\triangleright} V_{\nu}-\Gamma_{\mu \nu}^{\alpha} \star V_{\alpha} .
$$

To be a covariant derivative the connection has to transform as follows:

$$
\delta_{\xi}^{\star} \Gamma_{\mu \nu}^{\alpha}=-X_{\xi}^{\star} \stackrel{\star}{\triangleright} \Gamma_{\mu \nu}^{\alpha}-X_{\left(\partial_{\mu} \xi^{\rho}\right)}^{\star \star} \stackrel{\triangleright}{\triangleright} \Gamma_{\rho \nu}^{\alpha}-X_{\left(\partial_{\nu} \xi^{\rho}\right)}^{\star} \stackrel{\star}{\triangleright} \Gamma_{\mu \rho}^{\alpha}+X_{\left(\partial_{\rho} \xi^{\alpha}\right)}^{\star} \stackrel{\star}{\triangleright} \Gamma_{\mu \nu}^{\rho}-\partial_{\mu} \partial_{\nu} \xi^{\alpha} .
$$

This can easily be generalized to arbitrary tensor fields.

\subsection{Curvature and torsion}

The curvature and torsion tensors can be defined as usual

$$
\left[D_{\mu} \stackrel{\star}{,} D_{\nu}\right] \star V_{\rho}=R_{\mu \nu \rho}^{\sigma} \star V_{\sigma}+T_{\mu \nu}^{\alpha} \star D_{\alpha} V_{\rho} .
$$

They can be expressed in terms of the connection:

$$
\begin{aligned}
& R_{\mu \nu \rho}^{\sigma}=\partial_{\nu}^{\star} \stackrel{\star}{\triangleright} \Gamma_{\mu \rho}^{\sigma}-\partial_{\mu}^{\star} \stackrel{\triangleright}{\triangleright}^{\sigma} \Gamma_{\nu \rho}^{\sigma}+\Gamma_{\nu \rho}^{\beta} \star \Gamma_{\mu \beta}^{\sigma}-\Gamma_{\mu \rho}^{\beta} \star \Gamma_{\nu \beta}^{\sigma}, \\
& T_{\mu \nu}{ }^{\alpha}=\Gamma_{\nu \mu}^{\alpha}-\Gamma_{\mu \nu}^{\alpha} .
\end{aligned}
$$

From the transformation law of the connection (13) follows that curvature and tension transform like tensors if the deformed Leibniz rule (12) is used. 


\subsection{Metric tensor}

The relevant dynamical variable in gravity is the metric tensor. It is introduced as a covariant symmetric tensor of rank two:

$$
\delta_{\xi}^{\star} G_{\mu \nu}=-X_{\xi}^{\star} \stackrel{\star}{\triangleright} G_{\mu \nu}-X_{\left(\partial_{\mu} \xi^{\rho}\right)}^{\star} \stackrel{\star}{\triangleright} G_{\rho \nu}-X_{\left(\partial_{\nu} \xi^{\rho}\right)} \stackrel{\star}{\triangleright} G_{\mu \rho} .
$$

For $\theta=0$ we identify it with the usual metric field:

$$
\left.G_{\mu \nu}\right|_{\theta=0}=g_{\mu \nu}
$$

We will see that $g_{\mu \nu}$ is the only dynamical variable in the Einstein-Hilbert theory.

Next we have to construct the $\star$-inverse of the metric:

$$
G_{\mu \nu} \star G^{\nu \rho \star}=\delta_{\mu}^{\rho} .
$$

Let us first construct the $\star$-inverse of a function that is invertible in the undeformed algebra:

$$
f \cdot f^{-1}=1 \text {. }
$$

The star inverse $f^{-1 \star}$ is defined by

$$
f \star f^{-1 \star}=1 .
$$

It exists as a geometric series because $f^{-1}$ exists. The additional terms are a power series in $\theta$. To find $f^{-1 \star}$ in a compact version we start from

$$
\begin{aligned}
& f \star f^{-1}=1+\mathcal{O}(\theta), \\
& \left(f \star f^{-1}\right)^{-1 \star}=\left(1+f \star f^{-1}-1\right)^{-1 \star}=\sum_{n=0}^{\infty}\left(1-f \star f^{-1}\right)^{n \star} .
\end{aligned}
$$

The star at the $n$th power means that all the products are star products. By definition we know that

$$
\left(f \star f^{-1}\right) \star\left(f \star f^{-1}\right)^{-1 \star}=1 .
$$

The star multiplication is associative. We use this for equation (18) and write it in the form

$$
f \star\left(f^{-1} \star\left(f \star f^{-1}\right)^{-1 \star}\right)=1 .
$$

It follows that

$$
f^{-1 \star}=f^{-1} \star\left(f \star f^{-1}\right)^{-1 \star} .
$$

The factor $\left(f \star f^{-1}\right)^{-1 \star}$ has been calculated in (17) as a power series expansion in $f$ and $f^{-1}$. We insert this into (19) and find that $f^{-1 \star}$ can be expressed in $f$ and $f^{-1}$.

To invert the metric tensor we follow the analogous procedure.

$$
G_{\mu \nu} \cdot G^{\nu \rho}=\delta_{\mu}^{\rho}, \quad G_{\mu \nu} \star G^{\nu \rho \star}=\delta_{\mu}^{\rho}
$$

are the defining equations for $G^{\mu \nu}$ and $G^{\mu \nu \star}$. For $G^{\mu \nu \star}$ we find

$$
G^{\mu \nu \star}=G^{\mu \rho} \star\left(G \star G^{-1}\right)^{-1 \star}{ }_{\rho}^{\nu},
$$


where $G$ and $G^{-1}$ are short for the matrices $G_{\mu \nu}$ and $G^{\mu \nu}$ respectively. We also can show that

$$
\left(G \star G^{-1}\right)^{-1 \star}=\sum_{n \geq 0}\left(1-G \star G^{-1}\right)^{n \star} .
$$

Because the $\star$-product is not commutative $G^{\mu \nu \star}$ will be not symmetric in $\mu$ and $\nu$. Note that we always work with the formal power series expansions (in the sense that they do not necessarily converge), so the solutions for the inverses (19) and (20) are formal.

It can now be shown explicitly from the transformation law (15) for $G_{\mu \nu}$ that $G^{\mu \nu \star}$ transforms as a contravariant tensor of rank 2. Deformed Leibniz rule!

In formulating the ER theory we meet the determinant and the square root of the determinant. As it is more difficult to generalize the square root to a $\star$-square root we first introduce the vielbein as the "square" root of the metric tensor. It consists of four covariant vector fields $E_{\mu}{ }^{a}$ that form the metric:

$$
G_{\mu \nu}=\frac{1}{2}\left(E_{\mu}^{a} \star E_{\nu}^{b}+E_{\nu}^{a} \star E_{\mu}^{b}\right) \eta_{a b} .
$$

As the $\star$-product is noncommutative we have symmetrized $G_{\mu \nu}$ explicitly. For the vielbein fields we demand in analogy with (16)

$$
\left.E_{\mu}^{a}\right|_{\theta=0}=e_{\mu}^{a} .
$$

From the vector-like transformation properties of $E_{\mu}{ }^{a}$ follows that $G_{\mu \nu}$ transforms like a tensor. The determinant of the vielbein is defined as follows:

$$
E^{\star}=\operatorname{det}_{\star} E_{\mu}^{a}=\frac{1}{4 !} \varepsilon^{\mu_{1} \ldots \mu_{4}} \varepsilon_{a_{1} \ldots a_{4}} E_{\mu_{1}}^{a_{1}} \star \cdots \star E_{\mu_{4}}^{a_{4}} .
$$

The star on $E^{\star}$ and $\operatorname{det}_{\star}$ indicates that all the multiplications are $\star$-multiplications. This normalization was chosen such that

$$
\left.\operatorname{det}_{\star} E_{\mu}^{a}\right|_{\theta=0}=\operatorname{det}_{\mu}{ }^{a} \text {. }
$$

The second $\varepsilon$-tensor is necessary because the $\star$-product is noncommutative.

The important property of the determinant is that it transforms as a scalar density:

$$
\delta_{\xi}^{\star} E^{\star}=-X_{\xi}^{\star} \stackrel{\star}{\triangleright} E^{\star}-X_{\left(\partial_{\mu} \xi^{\mu}\right)}^{\star} \stackrel{\star}{\triangleright} E^{\star} .
$$

This is a consequence of the transformation law of the vielbein. This justifies the definition (21) of the determinant.

We now have all the ingredients we need to proceed for the formulation of the Einstein-Hilbert dynamics.

\subsection{Christoffel symbol}

We demand that the covariant derivative of $G_{\mu \nu}$ vanishes:

$$
D_{\alpha} G_{\beta \gamma}=\partial_{\alpha}^{\star} \stackrel{\star}{\triangleright} G_{\beta \gamma}-\Gamma_{\alpha \beta}^{\rho} \star G_{\rho \gamma}-\Gamma_{\alpha \gamma}^{\rho} \star G_{\beta \rho}=0 .
$$

We permute the indices, assume that $G_{\alpha \beta}$ is symmetric and obtain by following the analogous procedure in the classical case:

$$
\Gamma_{\alpha \beta}^{\sigma}=\frac{1}{2}\left(\partial_{\alpha}^{\star} \stackrel{\star}{\triangleright} G_{\beta \gamma}+\partial_{\beta}^{\star} \stackrel{\star}{\triangleright} G_{\alpha \gamma}-\partial_{\gamma}^{\star} \stackrel{\star}{\triangleright} G_{\alpha \beta}\right) \star G^{\gamma \sigma \star} .
$$

The connection is entirely expressed in terms of $G_{\alpha \beta}$. In this case we call the connection Christoffel symbol.

Again the transformation law of the connection (13) follows from the transformation law of $G_{\alpha \beta}$. 


\subsection{Ricci tensor and curvature scalar}

We obtain the Ricci tensor by contracting the upper index with one of the three lower indices of the curvature tensor (14). As the curvature tensor is antisymmetric in the first two indices we have only two choices left. Contracting of the second index is a deformation of the classical Ricci tensor:

$$
R_{\mu \nu}=R_{\mu \sigma \nu}{ }^{\sigma}
$$

The contraction $R_{\mu \nu \sigma}{ }^{\sigma}$ vanishes in the classical limit $\theta \rightarrow 0$. Nevertheless, we could add such a term to the Ricci tensor (23) and obtain a deformation of the classical Ricci tensor.

We see that the deformation of the classical theory is not unique. Terms that are covariant and vanish for $\theta \rightarrow 0$ are quite possible. To really make the deformation unique an additional requirement has to be added. We take simplicity and define the Ricci tensor by (23).

The curvature scalar we define by contraction with $G^{\mu \nu \star}$

$$
R=G^{\mu \nu \star} \star R_{\mu \nu}
$$

Again, as $G^{\mu \nu \star}$ is not symmetric this is a choice.

We can now show by starting from the tensor $G_{\mu \nu}$ that the curvature scalar transforms as a scalar field

$$
\delta_{\xi}^{\star} R=-X_{\xi}^{\star} \stackrel{\star}{\triangleright} R=-\xi^{\mu}\left(\partial_{\mu} R\right) .
$$

\subsection{Lagrangian}

The curvature scalar multiplied by the determinant $E^{\star}$ transforms like a scalar density. From (24) and (22) follows:

$$
\delta_{\xi}^{\star}\left(E^{\star} \star R\right)=-\partial_{\mu}^{\star} \stackrel{\star}{\triangleright}\left(X_{\xi^{\mu}}^{\star} \stackrel{\star}{\triangleright}\left(E^{\star} \star R\right)\right) .
$$

To define an action and the variational principle to find the field equation we have to give a definition for the integral. A possible definition is

$$
\int f=\int \mathrm{d}^{4} x f .
$$

This integral has the trace property

$$
\int \mathrm{d}^{4} x f \star g=\int \mathrm{d}^{4} x g \star f .
$$

A suitable action for a gravity theory on deformed spaces is:

$$
S_{\mathrm{EH}}=\frac{1}{2} \int \mathrm{d}^{4} x\left(E^{\star} \star R+\text { c.c. }\right) .
$$

A reminder: By all the transformation laws of products of fields the deformed Leibniz rule (12) has to be used.

\subsection{Field equations}

The trace property of the integral (25) allows us to define a variational principle. Vary one field after the other but always bring it first to the left (right) hand side of the integral by using the trace property. 
[1] Aschieri P., Blohmann C., Dimitrijević M., Meyer F., Schupp P., Wess J., A gravity theory on noncommutative spaces, Classical Quantum Gravity, 2005, V.22, 3511-3522, hep-th/0504183.

[2] Aschieri P., Dimitrijević M., Meyer F., Wess J., Noncommutative geometry and gravity, Classical Quantum Gravity, 2006, V.23, 1883-1912, hep-th/0510059.

[3] Bayen F., Flato M., Fronsdal C., Lichnerowicz A., Sternheimer D., Deformation theory and quantization. I. Deformations of symplectic structures, Ann. Physics, 1978, V.111, 61-110.

[4] Sternheimer D., Deformation quantization: twenty years after, AIP Conf. Proc., 1998, V.453, 107-145, math.QA/9809056.

[5] Kontsevich M., Deformation quantization of Poisson manifolds, Lett. Math. Phys., 2003, V.66, 157-216, q-alg/9709040.

[6] Waldman S., An introduction to deformation quantization, Lecture Notes, 2002, see http://idefix.physik.uni-freiburg.de/ stefan/Skripte/intro/index.html.

[7] Weyl H., Quantenmechanik und Gruppentheorie, Zeit. für Phys., 1927, V.46, 1-46.

[8] Moyal J.E., Quantum mechanics as a statistical theory, Proc. Cambridge Phil. Soc., 1949, V.45, 99-124.

[9] Wess J., Zumino B., Covariant differential calculus on the quantum hyperplane, Nuclear Phys. B Proc. Suppl., 1991, V.18, 302-312.

[10] Woronowic S.L., Differential calculus on compact matrix pseudogroups (quantum groups), Comm. Math. Phys., 1989, V.122, 125-170.

[11] Wess J., Deformed coordinate spaces; derivatives, in Proceedings of the BW2003 Workshop on Mathematical, Theoretical and Phenomenological Challenges Beyond the Standard Model: Perspectives of Balkans Collaboration (2003, Vrnjačka Banja, Serbia), Vrnjačka Banja, 2003, 122-128, hep-th/0408080.

[12] Wess J., Differential calculus and gauge transformations on a deformed space, hep-th/0607251.

[13] Wess J., Deformed gauge theories, hep-th/0608135.

[14] Aschieri P., Dimitrijević M., Meyer F., Schraml S., Wess J., Twisted gauge theories, Lett. Math. Phys., 2006, V.78, 61-71, hep-th/0603024.

[15] Vassilevich D.V., Twist to close, Modern Phys. Lett. A, 2006, V.21, 1279-1284, hep-th/0602185.

[16] Drinfel'd V.G., On constant quasiclassical solutions of the Yang-Baxter equations, Soviet Math. Dokl., 1983, V.28, 667-671.

[17] Reshetikhin N., Multiparameter quantum groups and twisted quasitriangular Hopf algebras, Lett. Math. Phys., 1990, V.20, 331-335.

[18] Gerstenhaber M., Giaquinto A., Schack S.D., Quantum symmetry, in Quantum Groups, Lecture Notes in Math., V.1510, Editor P.P. Kulish, Berlin, Springer-Verlag, 1992, 9-46.

[19] Kulish P.P., Lyakhovsky V.D., del Olmo M.A., Chains of twists for classical Lie algebras, J. Phys. A: Math. Phys., 1999, V.32, 8671-8684, math.QA/9908061. 